\title{
Una vez más invisibles en pandemia: salud mental, personas con discapacidad y pueblos originarios
}

\section{Once more invisible in pandemic: mental health, people with disabilities and originating peoples}

\author{
Patricia Cristaldo ${ }^{a}$, Rosa Ruffinellia , Sofía Gavilán ${ }^{a}$, Belinda García ${ }^{a}$, Álvaro \\ Lo Bianco ${ }^{a}$
}

\begin{abstract}
Resumen
Este ensayo problematiza la vulnerabilidad en la que se encuentra nuestra población ante la pandemia COVID-19, con un sistema de protección social casi nulo y un sistema de salud más que precario y deficiente. Se hizo un repaso por sectores históricamente desprotegidos como pueblos originarios o personas con discapacidad que una vez más fueron los excluidos, a partir de la recopilación de informaciones de periódicos que tímidamente fueron hablando de estos temas. Ante el aislamiento social obligatorio, la principal respuesta del estado para mitigar la crisis económica fue mediante transferencias monetarias, a sectores enmarcados en el trabajo informal. Si bien la transferencia monetaria fue necesaria, los organismos del estado no cuentan con un censo o registros que permitan asistir a estos sectores, por lo que la ejecución fue retrasada, desacertada, poco accesible para las personas y dejando al desnudo la improvisación, en la ejecución de la política pública. A partir de estas reflexiones sumamos las miradas y los análisis para complejizar la cuestión social, en tiempos de pandemia, evaluando en este breve ensayo las realidades de los sujetos sociales que están siendo atravesados por las distintas facetas de la COVID-19 y más aún aquellos grupos minoritarios históricamente invisivilizados.
\end{abstract}

Palabras clave: pandemia, vulnerable, estado. a Universidad Nacional de Asunción, Facultad de Ciencias Sociales, Paraguay.

Correspondencia a: rruffinelli @yahoo.es

Recibido:

20 de mayo de 2020

Aceptado:

26 de junio de 2020

Artículo publicado en acceso abierto bajo la Licencia Creative Commons.

(c) (i)

Cita:

Cristaldo, P., Ruffinelli, R. Gavilán, S., García, B., Lo Bianco, A.'(2020). Una vez más invisibles en pandemia: salud mental, personas con discapacidad y pueblos originarios. Kera Yvoty: reflexiones sobre la cuestión social, 5 (número especial), 86-92.

\begin{abstract}
This essay problematizes the vulnerability of our population to the COVID-19 pandemic, with an almost null social protection system and a health system that is more than precarious and deficient. A review was made by historically unprotected sectors such as indigenous peoples or people with disabilities who were once again the excluded, from the collection of information from newspapers that were timidly talking about these issues. Faced with compulsory social isolation, the main response of the state to mitigate the
\end{abstract}


economic crisis was through monetary transfers, to sectors framed in informal work. Although the monetary transfer was necessary, the state agencies do not have a census or records that allow them to assist these sectors, so the execution was delayed, inappropriate, not very accessible to people and leaving improvisation bare, in the execution of public policy. From these reflections we add the views and the analyzes to make the social question more complex, in times of pandemic, evaluating in this short essay the realities of the social subjects that are being crossed by the different facets of COVID-19 and even more so those historically unseen minority groups.

Keywords: pandemic, vulnerable, state.

\section{Introducción}

La pandemia denominada COVID-19 sorprendió al mundo desde hace unos pocos meses, con su vertiginosa expansión, la cual puso en evidencia la fragilidad de los mejores sistemas de salud y los mejores modelos de protección social a nivel mundial, dejando a países de primer mundo desbordados y sin respuestas rápidas como efectivas a su población.

Este escenario se agrava en aquellas economías que ya registraban altos niveles de endeudamiento y una proyección de menor crecimiento, como en muchos países de la región. Sin duda, los estados tienen que asumir ahora nuevas funciones económicas y sociales para proteger a la población, no solamente del contagio (y evitar así el colapso de los sistemas sanitarios), sino también hacer frente a la recesión de la actividad económica que ya se ha cobrado millones de puestos de trabajo en todo el mundo.

Esta revalorización del rol activo del Estado concierne, entonces, principalmente a su dimensión de cohesión social y de articulación del conjunto de las prácticas sociales. Es decir, a su capacidad para atender simultáneamente a la complejidad de cada instancia que lo compone, con sus tiempos y modos de intervención particulares (Gorriti, 2020).

En Paraguay, si bien hasta el momento las cifras de infectados y fallecidos se mantiene relativamente baja, en este corto tiempo se ha evidenciado un sinfín de debilidades en materia de protección social y la desprotección en las que se encuentran algunos sectores invisibles en la sociedad.

Este ensayo se encuentra enmarcado en la Resolución FACSO № 073/2020 abriendo el debate desde la academia sobre los efectos sociales de la pandemia. "La tarea fundamental de las Ciencias Sociales desde la academia, es mostrar la realidad social al descubierto, de modo a contribuir con insumos provenientes del estudio y análisis, para que, de un lado, las instancias responsables de generar respuestas a las necesidades y demandas sociales dispongan de estas (entre otras fuentes de estudio y diagnóstico), para abarcarlas".

\section{Contexto país, una mirada hacia la población en situación de vulnerabilidad, en cifras}

La situación de la población paraguaya, de antes de la pandemia conforme a la Encuesta Permanente de Hogares 2017, muestra un 26,40\% del total de habitantes del país en situación de pobreza, es decir, alrededor de 1 millón 809 mil personas residen en hogares cuyos ingresos per cápita son inferiores al costo de una canasta básica de consumo. En el área rural, la pobreza total afecta al 36,22\%, mientras que el área urbana presenta una menor proporción de habitantes viviendo en condiciones de pobreza (20,25\%). La población en situación de extrema pobreza, llega a 301 mil personas aproximadamente.

Respecto a la inversión social, nuestro país se mantiene entre los países con menor inversión social en la región, invirtiendo solo 147 dólares per cápita en servicios 
sociales al año, mientras que Argentina 1601 y Brasil 1419, siendo la recaudación tributaria de nuestro país apenas el $12 \%$ de PIB (Ramírez, 2016).

\section{Abandono, desprotección y vulnerabilidad}

La epidemia dejó al desnudo la vulnerabilidad en la que se encuentra nuestra población, con un sistema de protección social casi nulo y un sistema de salud más que precario y deficiente, principalmente en las áreas de prevención y contención en salud; el cual tuvo que ser equipado a medias, en un tiempo récord, para paliar la crisis, en medio de escándalos de corrupción.

En el marco del debate actual, sobre reforma del estado, reconocidos defensores del neoliberalismo como Amilcar Ferreira y Fernández Bogado plantean "achicar el estado", disminuyendo la cantidad de funcionarios públicos, proponiendo exonerar de impuestos a sectores (poderosos) con más ingresos económicos en nuestra economía, privatizar entidades públicas, utilizar los fondos jubilatorios, entre otros, para hacer frente a la pandemia.

Las necesidades del Paraguay son opuestas a lo que proponen los referentes neoliberales. Se necesita un Estado más eficiente, por lo que una reforma real del estado, debería implicar ciertos elementos como:

Una reforma del modelo de desarrollo que supere el modelo actual excluyente, reforma del sistema democrático, que incluya a gremios de sectores sociales históricamente excluidos en el diseño de políticas públicas, una reforma impositiva con una carga tributaria más justa y por sobre todo una profunda reforma del sistema de protección social.

Un nuevo sistema de protección social debe contar con datos exactos sobre la población paraguaya y sus diversas necesidades en la actualidad, en especial de los sectores más desprotegidos como son las familias de escasos recursos, las personas con discapacidad, los pueblos originarios y sobre ello construir políticas públicas eficientes, que superen el mero asistencialismo o las transferencias monetarias vacías de contenido superador.

\section{Reflexiones sobre la realidad de algunos grupos minoritarios en el contexto de la epidemia}

\subsection{La salud mental y pandemia COVID-19}

En el tiempo de la cuarentena total se ha podido observar que no se redujeron los casos de violencia intrafamiliar (Ultima Hora, 2020), tampoco bajaron los índices de maltrato y abuso infantil (ABC Color, 2020); todo esto nos lleva a la siguiente pregunta, ¿Qué políticas de salud mental se previeron, en relación a la disposición de cuarentena absoluta y aislamiento social?

Según la Organizacion Mundial de la Salud, OMS, "la salud es un estado completo de bienestar físico, mental y social y no solamente la ausencia de afecciones o enfermedades", poniendo énfasis con esto, al concepto de salud de manera integral, desde lo físico hasta lo psicosocial.

El sistema de salud pública en Paraguay, no sólo es deficiente en la prevención de enfermedades, también hace aguas en lo que refiere a la salud mental, teniendo como triste resultado, no solo los datos de maltrato o violencia, también una elevada tasa de suicidios (La Nación, 2019).

Igualmente se ha observado el impacto del confinamiento en los/as niños, niñas y adolescentes, con ataques de ira, irritabilidad, abulia, problemas de relacionamiento, entre otros; debido a que la mayoría no "estamos acostumbrados".

Lo vertiginosa que resultó ser la vida cotidiana, hace que no estemos acostumbrados a estar mucho tiempo en casa, a permanecer del tiempo allí, pues la mayor parte del día, padres y madres deben ir al (se va en el) trabajo, (debido a factores como el) para hacer frente al encarecimiento del costo de vida y la híper-explotación laboral, es por esto que en muchos casos el proceso de cuarentena, fue más difícil y traumático en diversa medida para la población. 
En el caso de los padres y madres, se han visto obligados a cumplir diferentes roles dentro de la cuarentena obligatoria como: ser maestros de escuela, dirigir actividades físicas y lúdicas, poner límites, contener emocionalmente a sus hijos/as; en el caso de las parejas, aprender a convivir más tiempo, sin que esto implique invadir la privacidad del otro; todo esto sin una sola política pública de orientación en salud mental, tips de relacionamiento o técnicas caseras de abordaje en situaciones de crisis.

Actualmente los dispositivos de salud mental existentes, tienen un rol pasivo, esperar a que el paciente consulte, esto conlleva dos problemas en sí; el primero que, entre el momento de la afección y la decisión de ir a consultar, hay una importante brecha de tiempo. Lo segundo, que una vez tomada la decisión de ir a consultar, la persona debe esperar meses, para conseguir un turno, a esto hay que sumarle que el enfoque de la mayoría de los tratamientos, no es integral y concentra mucho el proceso en suministrar psicofármacos, como lo ha señalado el Dr. Carlos Portillo, en su conferencia, sobre "Salud Mental en tiempos de Pandemia de COVID-19" (Portillo, 2020).

\subsection{Personas con discapacidad y pandemia COVID-19}

Otro sector extremadamente desprotegido históricamente y también durante este proceso han sido las personas con discapacidad, lo cual constituye el $20 \%$ de la población paraguaya (JICA, 2012), y que carece de condiciones básicas para su desarrollo, debido a las barreras impuestas por la sociedad más que por su propia condición tales como el bajo nivel de escolaridad y el escaso ingreso al mercado laboral.

La sociedad sigue requiriendo de las mujeres que asuman el cuidado de personas mayores, enfermas o dependientes, de los niños y las niñas, y que compaginen estos cuidados con su actividad laboral. Esta sobre exigencia tiene consecuencias en la salud y en la calidad de vida de las cuidadoras, sobre todo en estos momentos de encierro, en donde ni siquiera hay posibilidad de delegar la tarea en terceros.

Como señala (Dakduk et al., 2010, p. 417) cuando se habla de familias cuidadoras realmente nos estamos refiriendo a las mujeres, pues existe la creencia que el "cuidado" es trabajo femenino, actividad que señala no sólo a la esposa o hijas, sino también a nueras, nietas, sobrinas, ahijadas; pues ser mujer es suficiente requisito para asumir el compromiso de cuidar.

Como si estas condiciones no fueran suficientemente catastróficas, la OMS informa que "El impacto de esta enfermedad podría ser mayor en ciertos grupos de la población, como las personas con discapacidad, y que la única manera de mitigar si los principales interesados adoptan medidas de protección apropiadas".

Por otra parte, el distanciamiento social para las personas con discapacidad dependientes, tiene una connotación particular "las medidas de contención, como el distanciamiento social y el aislamiento personal, pueden ser imposibles para quienes requieren apoyo para comer, vestirse o ducharse" como menciona la Relatora Especial sobre los derechos de personas con discapacidad de las Naciones Unidas (OACNUDH, 2020).

Es entonces donde nos preguntamos, en las medidas tomadas para prevenir el contagio, son considerados las diversidades, las minoríasque no respondena estereotipos predominantes como quedan, sujetos a su suerte, al margen de las políticas públicas, una vez más invisible.

\subsection{Pueblos Originarios y pandemia COVID-19}

Y haciendo una mirada a la región occidental podemos ver una realidad, con otras características, hablamos de los pueblos originarios, otra minoría que tampoco formó parte de las prioridades y con postergaciones históricas.

En las comunidades indígenas del Chaco donde en la mayoría de ellas no existen centros de salud con medicamentos, 
ni terapia intensiva y mucho menos una ambulancia o móvil para trasladar a un paciente a otros centros asistenciales $y$ cuentan con un solo médico, situaciones que agudizan el miedo en los pobladores

"Por eso tenemos mucho miedo de que el virus llegue a la comunidad". (Entrevista a líder indígena, 2020)

Por otro lado, no cuentan con agua corriente para cumplir con las medidas del lavado de las manos en forma constante, se las arreglan con agua de tajamar o de las lluvias. El seguro de salud, solo lo tienen aquellos que trabajan en los supermercados de la Cooperativa Chortizer, para ser asistidos en el Centro de Salud.

Desde la cuarentena, pocos son los que salen a cumplir sus labores. Los líderes decidieron bloquear los accesos de sus pueblos para evitar que el virus llegue a sus localidades. Se organizan en guardias que van rotando en diferentes horarios. En la mayoría de las comunidades ya se siente la falta de alimentos la gente está "paleando", el "menona" nos está provisionando de alimentos, porque esperar el kit del gobierno tardará mucho.

"Una lástima, el gobierno promete, pero no cumple, mucho más a los pueblos indígenas, la ayuda tarda en llegar". (Entrevista a líder indígena, 2020)

Las mismas necesidades y urgencias se sienten en el Pueblo Nivaclé, de Boquerón, señala la lideresa Floris Yegros, "desde la Municipalidad se aprobaron 26o millones de guaraníes para la compra de kits de alimentos, para las familias, pero aún no recibieron la asistencia por parte de la comuna”. Además, manifiesta que los indígenas de Yalve Sanga tienen que pagar la tarifa de la electricidad, desconocen si se les exonerará el pago o se les acumulará el gasto por la energía eléctrica.

Ante el avance de la COVID-19 en el país, los indígenas se encuentran en una doble vulnerabilidad; no tienen a disposición centros asistenciales, ni la garantía del traslado de pacientes si llegara a "entrar la enfermedad"; han acatado las medidas sanitarias impuestas de aislamiento, aguardando la asistencia comprometida del Estado en materia de alimentación, medicamentos y agua. Miles de indígenas en el Paraguay están pasando hambre y abandono, precarizando aún más sus condiciones de vida, que ya de por sí, antes de la llegada del COVID-19 eran difíciles. Datos proveídos por el propio Estado señalan que poco más del $65 \%$ de miembros de los Pueblos Indígenas se encuentra en situación de pobreza y más del $30 \%$ en pobreza extrema (DGEEC, 2017). La histórica falta de concreción de derechos a los Pueblos Indígenas, así como del despojo territorial del cual nunca han sido reparados de forma integral, a través de la restitución de sus tierras y de la implementación de proyectos de desarrollo que les permitan subsistir enteramente por sus propios medios.

\section{Conclusiones}

Lo evidenciado durante la pandemia, no son situaciones nuevas sino la forma común en que el estado paraguayo responde a las necesidades de los grupos vulnerables en general y específicamente aquellos mencionados en este ensayo; personas con discapacidad, pueblos originarios y atención a la salud mental, lo cual evidentemente requiere de una profunda reforma del estado, estableciendo políticas públicas que apunten a mejorar sustancialmente, la calidad de vida de los ciudadanos, interrumpiendo la reproducción de la pobreza a gran escala.

Inclusive referentes del neoliberalismo como Fernández, Giménez y Cano (2017) en su análisis de la realidad sostienen que:

No es posible que casi un tercio de la población vaya a la cama sin haber comido nada. Es ofensivo observarque el Paraguay que puede dar de comer a 60 millones de seres humanos no lo pueda hacer con el 3\% de esa cantidad, 
no podemos ser tan eficientes vacunando 14 millones de vacas y no lo podamos hacer ni con la mitad de nuestros niños. (p. 9)

Si entendemos la salud como un todo, es necesario re-diseñar de manera urgente las políticas de salud, enfocadas en la prevención real, no solo desde campañas publicitarias, sino con profesionales en todas las áreas, especialmente psiquiatras, psicólogos, enfermeros, médicos especializados, trabajadores sociales, integrados en equipos de trabajo multidisciplinarios, como en algún momento fueron pensadas las APS (Atención primaria en salud).

Estos equipos multidisciplinarios, deben llegar a las comunidades, a los barrios, recabando datos, que nos permitan tener un panorama más claro, de la situación real en cuanto al estado de salud de la gente, mediante estrategias de abordajes familiares en todos los ámbitos de la salud, que funcionen como dispositivos de contención para futuras crisis.

Porotrolado, tambiénseríaimportante pensar en espacios colectivos de integración y promoción de la salud mental, desde los diferentes organismos gubernamentales, municipalidad, gobernaciones, estado central, espacios libres de violencia y confortables, que actualmente no existen por sobre todo para poblaciones en probable situación de vulnerabilidad, como niños, mujeres, adultos mayores, indígenas. Estos espacios deberían tener por un lado elementos lúdicos, artísticos que permiten la expresividad de las emociones, y, por otro lado, herramientas terapéuticas, como terapias ocupacionales, actividades de reflexión grupal, entre otros.

El mundo ha cambiado, "se escucha decir frecuentemente", sin embargo, depende de nosotros como profesionales y futuros profesionales, que cambie para bien y por sobre todo que esos cambios contemplen cosas que normalmente son ninguneadas, como la salud mental.

La universidad y la Facultad de
Ciencias Sociales, tienen un rol central en el actual debate sobre la reforma del estado, aportando desde la academia un criterio de historicidad, sobre las políticas públicas a la luz de los hechos y su eficiencia o fracaso. A su vez, generando espacios de debate y reflexión, con todos los actores de la comunidad académica.

Los efectos sociales de la pandemia COVID-19 deben ser temas de investigación impulsados desde la Facultad de Ciencias Sociales, investigaciones que permitan no solo la visibilización de los sectores históricamente invisibilizados, sino a su vez generen propuesta de políticas públicas que respondan a las necesidades reales de los mismos.

La Facultada de Ciencias Sociales se caracteriza por el contacto directo con las comunidades, su forma ver y entender la realidad, sus fortalezas y debilidades, su historia y sus tradiciones, hecho que posibilita contar con herramientas más efectivas para la investigación y análisis de estas realidades y por ende generar propuestas de políticas públicas de impacto real hacia la población.

Finalmente podemos concluir que desde las ciencias sociales sumamos las miradas y los análisis para complejizar la cuestión social, en tiempos de pandemia, analizando en este breve ensayo las realidades de los sujetos sociales que están siendo atravesados por las distintas facetas de la COVID-19 y más aún aquellos grupos minoritarios históricamenteinvisivilizados.

En tiempos de pandemia, necesitamos que las ciencias sociales alcen su voz más fuerte que nunca, exigiendo el cese de la corrupción, el acceso a derechos históricamente negados, que las ciencias sociales converjan a los movimientos sociales estableciendo una agenda común que persiga el bienestar de sociedad.

\section{Referencias}

ABC Color. (26 de abril de 2020). En tiempos de coronavirus se registran altas cifras de casos de maltrato infantil. https:// www.abc.com.py/edicion-impresa/ 
sociales/2020/04/26/en-tiempos-decoronavirus-se-registran-altas-cifrasde-casos-de-maltrato-infantil/

Dakduk, S., Ganzález, M., \& Malave,J. (2010). Percepciones a cerca de la pobreza y los pobres. Revista Latinoamericana de Psicología, 413/425.

Fernández, B., Giménez, F., \& Cano, R. (2017). Shock Educativo. Editorial Libre.

Gorriti, J. (2020). El estado de la pandemia. Teoria \& Cambio Social(1), 42-45.

JICA. (2012). Estudio de Prevalencia de Discapacidad en la Región Oriental del Paraguay. DELTA Consultora Integral .

La Nación. (7 de Junio de 2019). En Paraguay una persona se suicida por día. https://www.lanacion.com.py/ pais/2019/o6/o7/en-paraguay-unapersona-se-suicida-por-dia/

Moffat, A. (s.f.). Terapia de crisis.

OACNUDH, A. C. (marzo de 2020). Comunicado de prensa COVID-19: ¿Quién protege a las personas con discapacidad?, alerta experta de la ONU. https://oacnudh.hn/covid-19quien-protege-a-las-personas-condiscapacidad-alerta-experta-de-la-onu /?fbclid=IwAR22dlICZ7oWcGoywoEly dttGFMGa2Rif9UnpI7tQiYeJA_5XTn1x UwuolE

Organización Mundial de la Salud. (2020). Policía registra aumento de violencia familiar en cuarentena.

Organización Panamericana de la Salud. (abril de 2020). Consideraciones relativas a la discapacidad durante el brote de COVID-19. https://iris.paho. org/handle/10665.2/52026

Portillo, C. (abril de 2020). Salud Mental en tiempo de Pandemia. Ciclo de Charlas FACSO. Paraguay.

Ultima Hora. (20 de marzo de 2020). Policía registra aumento de violencia familiar en cuarentena. https:// www.ultimahora.com/policiaregistra-aumento-violencia-familiarcuarentena-n2876484.html

\section{Sobre los Autores}

\section{Patricia Cristaldo}

Trabajadora Social, Especialista en Planificación Estratégica Institucional, parte de equipo técnico de la Dirección de Planificación FACSO-UNA. Especialista en formulación, gestión y evaluación de proyectos sociales.

\section{Rosa Rufffinelli}

Doctoranda en Educación, UNA, Master en Trabajo Social, Licenciada en Trabajo Social por la UNA, Especialista en Educación y Rehabilitación con Enfoque Biopsicosocial, Docente Investigador de la UNA/FACSO, docente de la UNA/FENOB. Miembro del GT CLASCO Estudios Críticos en Discapacidad.

Sofía Gavilán

Trabajadora Social, egresa de la UNA. Especializada en Trabajos Comunitarios con Pueblos Originarios y campesino/as.

\section{Belinda García}

Trabajadora social. Investigadora en estudios de género.

Álvaro Lo Bianco

Docente, Psicólogo, terapeuta infantil. Especialista en Políticas Públicas para la Infancia y Adolescencia. Coordinador en el Ministerio de la Niñez y la Adolescencia. 